\title{
Exploring the Use of Proper Orthogonal Decomposition for Enhancing Blood Flow Images Via Computational Fluid Dynamics
}

\author{
R.H.P. McGregor ${ }^{1}$, D. Szczerba ${ }^{1}$, M. von Siebenthal ${ }^{1}$, K. Muralidhar ${ }^{2}$, \\ and G. Székely ${ }^{1}$ \\ ${ }^{1}$ Computer Vision Laboratory, Department of Electrical Engineering, ETH, 8092 \\ Zürich, Switzerland \\ 2 Department of Mechanical Engineering, Indian Institute of Technology Kanpur, \\ Kanpur 208 016, UP, India
}

\begin{abstract}
Obtaining high quality patient-specific flow velocity information is not an easy task. Available clinical data are usually poorly resolved and contain a significant amount of noise. We propose a novel approach to integrate computational fluid dynamics with measurement data to overcome this difficulty. By performing a proper orthogonal decomposition of simulated blood flow patterns for a given vascular location with various anatomical configurations it is possible to obtain a basis model for flow reconstruction. This is used to interpolate imaging data intelligently without having to perform a full flow simulation for each individual patient. This work focuses on assessing the feasibility of such a method.
\end{abstract}

\section{Introduction}

Obtaining accurate patient-specific blood flow information is essential for understanding cardio-vascular pathologies and predicting their onset [1]. For example, in the case of abdominal aortic aneurysms there is believed to be a significant correlation between flow related parameters, in particular the Wall Shear Stress (WSS) and the development of the disease. To date there are no clinical tools available for efficiently measuring these complex flow patterns in-vivo. Recently, Phase Contrast Magnetic Resonance Imaging (PC-MRI) has gained popularity as it is able to measure time-resolved three dimensional flow fields non-invasively 2. However, it has limited resolution and suffers under low signal to noise ratio, in particular at low flow velocities. To overcome these limitations it has been proposed to use Computational Fluid Dynamics (CFD) 3] in conjunction with medical imaging so as to obtain highly resolved time-dependent flow fields. This offers many advantages as it gives access to a wealth of data, not only velocity but also pressure distributions as well as secondary flow properties, such as WSS, which can easily be estimated by post-processing. Unfortunately such simulations require considerable computational effort. Another drawback is that this approach does not integrate much flow information from the measurements. 
Typically the only flow data which are taken into account are the mass flow rate at the inlets and outlets [4, the flow velocity distribution at the inflow and mass flow at the outlet [5] or even just an assumed flow waveform applied on the patient-specific geometry [6]. Using mass flow rate and a generic inflow velocity distribution completely ignores the effect of upstream disturbances and using a velocity field directly from the measurement means plugging any measurement error directly into the simulation.

We propose a novel approach to the problem of integrating CFD with PC-MRI measurements by performing all the simulation work beforehand to accumulate knowledge about the flow patterns typically occurring in a given vascular location. This is then used to perform an intelligent interpolation based on the measurement data. We perform a Proper Orthogonal Decomposition (POD) of a set of simulated flow fields and then look for the modal reconstruction which best fits the measurement data. This has the dual advantage of not requiring any patient-specific simulation and integrating a maximal amount of measured flow information into the final result, while still using the CFD solutions to reconstruct the fine detail. The method we use is based on Blanz and Vetter's work [7, which was originally developed for shape prediction from partial information and incorporates the treatment of noise in the measurement data.

POD, known in other fields as Principal Component Analysis or KarhunenLoève Transform is a widely used tool in Fluid Dynamics. Its main applications are for turbulence analysis 8] or model order reduction 9]. Recently there has been work on gappy data reconstruction using POD [10], where a full flow field is recovered from partial information, but without using a database and therefore not suitable for recovering a full flow field from very sparse data. In experimental fluid dynamics POD is often used, e.g., for data filtering, but to our knowledge it has never been used the way we propose here. In the field of biomedical imaging POD is used in statistical shape models for predicting organ motion [1] or model-based segmentation [12]. In comparison to the existing work our method brings two major novelties. Firstly, applying these methods to flow fields is a nontrivial step as these are non-linear and significantly less well behaved than the vector fields occurring in other applications; secondly, all the previous methods use measured data to build their database, whereas we utilize simulation results.

\section{Methods}

Our approach relies on collecting a large number of patient-specific vascular geometries and determining possible flow fields by CFD calculations, based on a representative, parameterized set of boundary conditions. We focus on the particular example of blood flow in the abdominal aortic bifurcation, which is of significant medical relevance as it is a determining factor in the pathogenesis of abdominal aortic aneurysms, a leading cause of death in developed countries. It also has the advantage of being a large geometry with fast flowing blood, making it a good example to assess the performance of our approach. Because this work aims to demonstrate feasibility rather than implement and validate 
a new method, we chose to use a $2 \mathrm{D}$ geometry, so as to test our assumptions on a simplified and well-defined problem and also to increase the amount of full time-dependent simulations we are able to perform. The key to our method is a database of trusted solutions. For the sake of this feasibility study we replace the representative collection of real patient anatomies with systematically generated 2D bifurcation geometries.

Generating the set of sample meshes. We started by constructing a reference mesh of a bifurcation. We then created several other meshes by changing the left and right branch take-off angles and adding more or less curvature to the reference mesh. These parameters were normally distributed within the approximate range of their natural variability as determined by Sun et al.[13], corresponding to a statistical variation of the bifurcation shape in real patients. The meshes all have 13'191 nodes and 25'682 cells.

Flow simulations. We then assigned different inflow boundary conditions to each mesh, these were parameterised using 3 variables: amplitude, flow frequency and flow asymmetry. The flow parameters were distributed in the same fashion as the geometric ones. In this way we generated several different flow problems, depending on 3 randomly distributed geometry parameters and 3 randomly distributed flow parameters, the mean values and standard deviations of which can be seen in figure 1. The simulations were performed by a Finite Element Method (FEM) code, solving the incompressible Navier-Stokes equations. The flow was assumed to be laminar, incompressible and Newtonian, with a density of 1020 $\mathrm{kg} / \mathrm{m}^{3}$ and a dynamic viscosity of $0.003 \mathrm{~Pa} / \mathrm{s}$. A time-dependent velocity profile was imposed at the inlet and the pressure was fixed at both outlets. The simulations were all performed for 5 heart cycles, the solution being taken at 100 evenly spaced timesteps in the last cycle. This resulted in a database corresponding to a set of simulations performed for several patient specific cases. Even if relying on artificial geometries, the key assumption at this point remains that the anatomies considered are trusted and representative for a larger population. We will refer to these database entries as the 'real' flow fields throughout the paper.

Coordinate transform. In order to make the simulated data comparable between the individual models, a common coordinate system is needed. As all geometries are deformed versions of the same mesh, the nodal correspondences are trivial. However, for the $x$ and $y$ velocity components to be comparable they need to be mapped onto a locally adapted coordinate system. In this $2 \mathrm{D}$ case, this was generated by solving two diffusion problems, one for the $x$ components of the edge tangents and one for the $y$ components. These are applied as Dirichlet boundary conditions on the flow domain and the diffusion equation is solved on the inside. The resulting two scalar fields are then used as the components of a smooth body-fitting vector field, as can be seen in figure 2 . This is used to define a locally adapted coordinate system. 


\begin{tabular}{|l|c|c|}
\hline Parameter & $\mu$ & $\sigma$ \\
\hline Right Branching Angle $\left.{ }^{\circ}\right]$ & 158.5 & 5.2 \\
Left Branching Angle $\left[^{\circ}\right]$ & 156.3 & 7.5 \\
Geometry Curvature $\left[^{\circ}\right]$ & 0 & 5 \\
\hline Flow Amplitude $[\mathrm{m} / \mathrm{s}]$ & 0.7 & 0.1 \\
Flow Frequency $[1 / \mathrm{s}]$ & 1.333 & 0.25 \\
Inflow Asymmetry & 0 & 0.35 \\
\hline
\end{tabular}

Fig. 1. Mean values $(\mu)$ and standard deviations $(\sigma)$ of the 6 parameters used to generate the various simulations

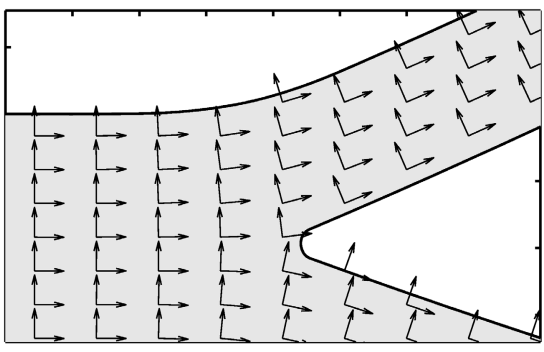

Fig. 2. Body-fitting coordinate system

Simulating an MRI Measurement. To generate pseudo-MRI measurements for our simulated flow fields in 2D, we create one image per timestep by averaging the velocity values inside each measurement pixel defined according to a realistic acquisition protocol, thus simulating the partial volume effect. We chose a $y$ (inplane) resolution of $d y=1.29[\mathrm{~mm}]$ and an $x$ (out-of-plane) resolution of $d x=$ $5[\mathrm{~mm}]$, corresponding to the usual anisotropy of the acquisition. The temporal resolution was reduced to 20 timesteps. The measurement error was taken to be $5 \%$ of the maximal absolute value of $u$ and $v$, as given in the literature [14] and modelled as white Gaussian noise. As a consequence, the absolute amount of noise is different in $x$ and $y$ direction, which is typical for PC-MRI. In the rest of the paper we will refer to these data as 'measured'.

Proper Orthogonal Decomposition and Flow Field Reconstruction. Each simulation was characterized by a representative area of interest, covering approximately 3 radii up and downstream from the bifurcation apex. Both $u$ and $v$, the $x$ and $y$ velocity components at all timesteps from the simulations are converted into the body-fitted coordinate system and concatenated to a sample vector $\mathbf{x}_{i}$. Each vector is then added to the sample matrix $\mathbf{X}=\left(\mathbf{x}_{1}, \mathbf{x}_{2}, \ldots, \mathbf{x}_{m}\right)$. Given a measurement $\mathbf{r} \in \mathbb{R}^{l}$, we are looking for the full-size solution vector $\mathbf{x} \in \mathbb{R}^{n}$, $l<n$, which best approximates our measurement. The method we rely on was proposed by Blanz and Vetter [7], which we shortly summarize here. We are looking for the solution $\mathbf{x}$ which minimises

$$
E=\|\mathbf{L x}-\mathbf{r}\|^{2}
$$

where $\mathbf{L}$ is a mapping $\mathbf{L}: \mathbb{R}^{n} \rightarrow \mathbb{R}^{l}$. A POD of $\mathbf{X}$ is then performed and we look for $\mathbf{c}$, the phase space coefficients which minimise $E$.

Additionally Blanz and Vetter extended the cost function with an additional term, which allows one to take the uncertainty of the measurements into account. This is based on the assumption that our measurement $\mathbf{r}$ is subject to an uncorrelated Gaussian noise with a variance $\sigma_{N}^{2}$. This was taken to be $5 \%$, the accepted error for PC-MRI [14]. This results in a combined cost function,

$$
E=\|\mathbf{Q} \mathbf{c}-\mathbf{r}\|^{2}+\sigma_{N}^{2} \cdot\|\mathbf{c}\|^{2}
$$


which reduces the strength of the constraints imposed by each measured value. $\mathbf{Q}$ is composed of the reduced set of the scaled eigenvectors of $\mathbf{X}$. For more details on how this cost function is built and then minimized refer to 7 .

\section{Results}

A total of 43 simulations were performed, each with different parameters, as described in section 2, The results from a subset of these are shown in figure 5 , or in more detail in figure $7(\mathrm{c})$.

To test the accuracy of our reconstructions we performed a series of leaveone-out tests. A pseudo-MRI measurement is generated for the left-out example, such as can be seen in figure 7(a). We build our database from the remaining simulations (42 samples) and then try to reconstruct the original flow data from the measurement and the database. The outcome of such an enhanced flow field can be seen in figure $7(\mathrm{~b})$. Figures 8(a) and 8(b) show the flow perpendicular to two different cross-sections. It can be seen that the recirculations are well captured despite the low velocities and correspondingly high noise levels. Not only does the method capture the flow structures common to all simulations, such as the flow recirculation in front of the apex, but also those which are much more geometry dependent such as the recirculation upstream of the lower branch, as seen in the bottom left corner of $7(\mathrm{c})$ and $7(\mathrm{~b})$. This demonstrates the method's ability for recovering flow detail invisible in the measurement (see figure 7(a) from the precomputed CFD solutions.

Figures 3 and 4 show the compactness and generalization ability of the model, which are the standard tools for evaluating the quality of a statistical shape model. Compactness $(C(M))$ is the sum of the model's eigenvalues from 1 to $M$ expressed as a fraction of their total sum. Generalization $(G(M))$ is obtained by doing leave-one-out tests for all simulations in the database, using only $M$ samples for reconstruction. The mean reconstruction error across all simulations, expressed as the sum of squared differences between estimated and real flow fields, is then plotted along with its standard deviation. For a detailed description of statistical model characterization, please refer to e.g. Styner et al. 15. As shown in figure 3, the first 17 modes already contain $98 \%$ of the model's variance. It may be seen on figure 4 that the achievable accuracy stabilizes after using approximately 15 modes.

We further investigated the number of MRI slices needed to achieve reasonable accuracy. The results of this study are depicted in figure 6. The first slice used was the one closest to the inlet, the second was the closest to the outlet and the following ones were further interleaved in this fashion. The reconstruction error $\epsilon$ is defined as the 4-norm of the difference between the estimated and the real flow fields, as the more common 2-norm did not account properly for localized errors. Interestingly, the reconstruction quality is better using a single slice than using two. This is because the model's fit close to the inlet (where slice 1 is taken) has a much higher influence on accuracy than close to the outlet (corresponding to slice 2), due to higher flow velocity. 


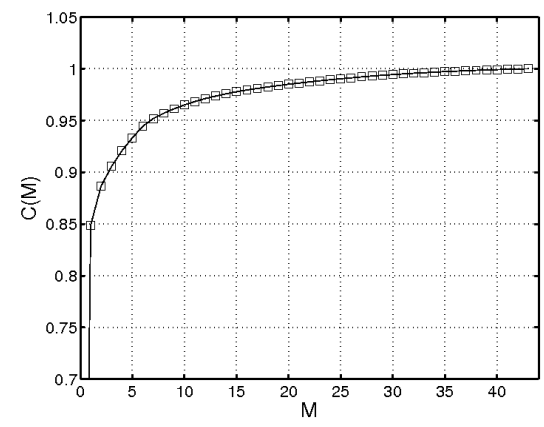

Fig. 3. Compactness of the model

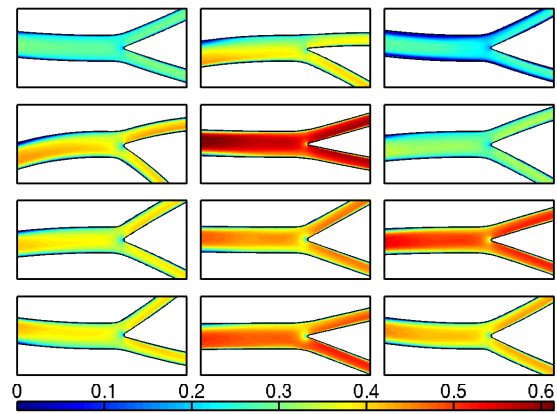

Fig. 5. The norm of velocity from the first 12 simulations at peak systole in $[\mathrm{m} / \mathrm{s}]$

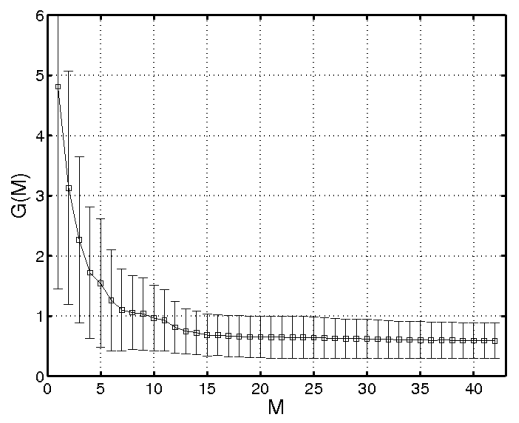

Fig. 4. Generalization ability of the model

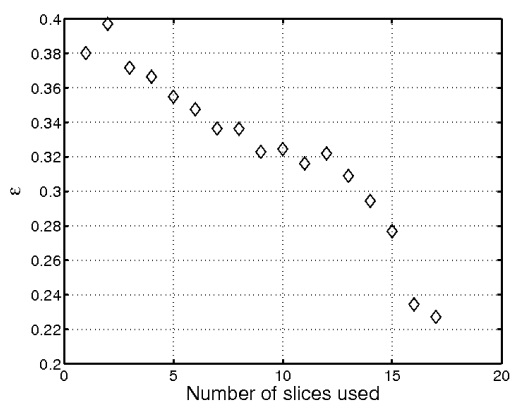

Fig. 6. Reconstruction error in function of the number of slices used from the measurement

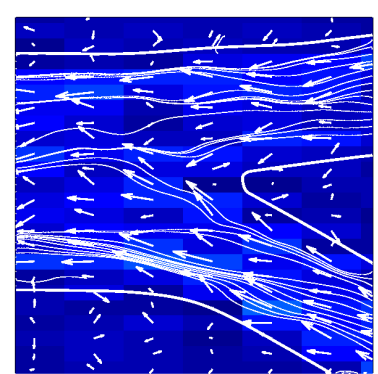

(a) Measured.

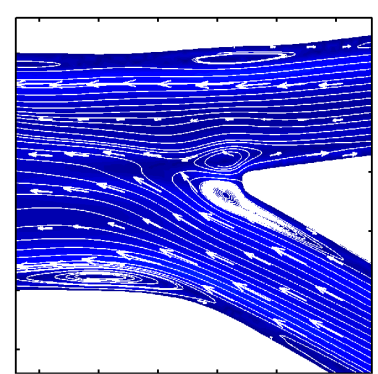

(b) Enhanced.

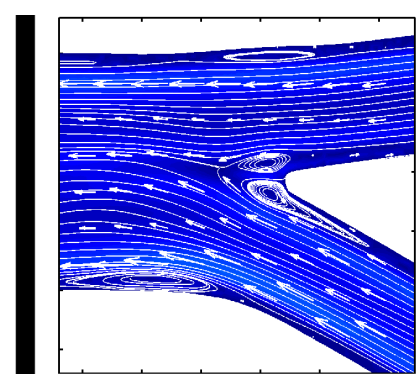

(c) Real.

Fig. 7. Flow field at end diastole showing recirculation zones

From the point of view of computational effort, the method is highly successful. Indeed, a full flow simulation took $1891[s]$ on our 64 -bit Intel ${ }^{\circledR}$ Xeon ${ }^{\circledR} 8$ core $2.66 \mathrm{GHz}$ machine with 16GB RAM when using an optimised commercial 


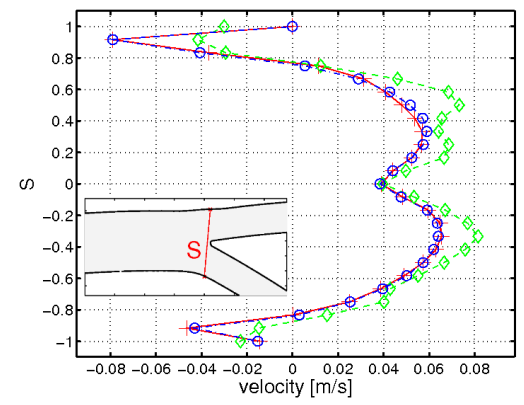

(a) Before the bifurcation.

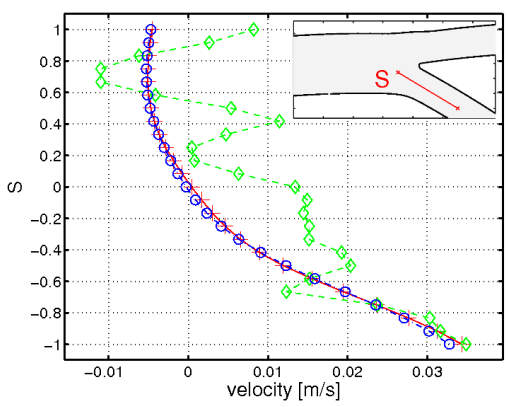

(b) Down the right branch.

Fig. 8. Velocity perpendicular to line $\mathrm{S}$ at mid diastole. + : real, $\diamond$ : measured, $\circ$ : enhanced.

code, whereas the flow field reconstruction using our method only took $48[s]$, making it roughly $40 \times$ faster.

\section{Discussion and Outlook}

From a fluid-mechanical point of view the major drawback of the method is that the estimated flow field does not solve the Navier-Stokes equations. However, for the physician, interested in the general flow patterns and the WSS distribution, this detail is irrelevant and justifies our use of a statistics based model instead of a physics based one. However, should one need a Navier-Stokes solution, it remains possible to perform a full simulation, using boundary conditions taken from the estimated flow field. It should be noticed that the no-slip boundary conditions are preserved as all the sample simulations fulfill them.

We have shown that our method provides good flow field estimates based on noisy, poorly resolved measurement data. This is a very encouraging result, which leads to believe that such a method could be used for enhancing PCMRI images based on precomputed CFD solutions. However there are many issues which will have to be considered before going on to a full 3D model, based on real MRI data. Most significantly, the simulations needed to populate the database would take considerable time and effort. They would each have to be based on separate, patient specific datasets and accurately capture the real flow distributions. It is, however, important to realize that the complexity of the simulations has no effect on the efficiency and speed of the reconstruction, so one could for example use Fluid Structure Interaction (FSI) simulations to capture the wall motion as well. The mesh generation and coordinate transform would become more complex and require some form of non-rigid registration to meaningfully map various geometries onto a reference. When confronted with clinical data, which are usually pathological, the amount of modes necessary to reconstruct a given case will increase and it might even occur that a measured flow field lies completely outside the convex hull of the database. Should this 
occur, that particular flow field should simply be simulated itself and added to the database, thus covering similar cases in the future. But basically these problems do not affect the fundamental reconstruction algorithm and we envision that one day such a method might be used to quickly obtain a highly-resolved flow field from an MRI measurement without going through all the hassle of dedicated numerical simulation, even if much work still remains before such an approach can be integrated into the daily clinical practice.

Acknowledgments. This work was supported by the Indo-Swiss Joint Research Programme.

\section{References}

1. Taylor, C.A., Draney, M.T.: Experimental and computational methods in cardiovascular fluid mechanics. Annual Review of Fluid Mechanics 36(1), 197-231 (2004)

2. Maier, S., et al.: Human abdominal aorta: comparative measurements of blood flow with $\mathrm{mr}$ imaging and multigated doppler us. Radiology 171(2), 487-492 (1989)

3. Steinman, D.A.: Image-based computational fluid dynamics modeling in realistic arterial geometries. Annals of Biomedical Engineering V 30(4), 483-497 (2002)

4. Milner, J.S., et al.: Hemodynamics of human carotid artery bifurcations: Computational studies with models reconstructed from magnetic resonance imaging of normal subjects. Journal of Vascular Surgery 28, 143-156 (1998)

5. Long, Q., et al.: Reconstruction of blood flow patterns in a human carotid bifurcation: A combined CFD and MRI study. Journal of Magnetic Resonance Imaging 11(3), 299-311 (2000)

6. Moore, J.A., et al.: Computational blood flow modeling based on in vivo measurements. Annals of Biomedical Engineering V27(5), 627-640 (1999)

7. Blanz, V., Vetter, T.: Reconstructing the complete 3D shape of faces from partial information. Informationstechnik und Technische Informatik 44(6), 295-302 (2002)

8. Berkooz, G., et al.: The proper orthogonal decomposition in the analysis of turbulent flows. Annual Review of Fluid Mechanics 25(1), 539-575 (1993)

9. Dowell, E.H.: Eigenmode analysis in unsteady aerodynamics - reduced-order models. AIAA Journal 34(8), 2545-2557 (1996)

10. Venturi, D., Karniadakis, G.: Gappy data and reconstruction procedures for flow past a cylinder. Journal of Fluid Mechanics 519, 315-336 (2004)

11. von Siebenthal, M., et al.: Inter-subject modelling of liver deformation during radiation therapy. In: Ayache, N., Ourselin, S., Maeder, A. (eds.) MICCAI 2007, Part I. LNCS, vol. 4791, pp. 659-666. Springer, Heidelberg (2007)

12. Hug, J., et al.: Model-based initialisation for segmentation. In: Computer Vision ECCV, pp. 290-306 (2000)

13. Sun, H., et al.: Measurement of the geometric parameters of the aortic bifurcation from magnetic resonance images. Annals of Biomedical Engineering 22(3), 229-239 (1994)

14. Frayne, R., et al.: Accuracy of $\mathrm{mr}$ phase contrast velocity measurements for unsteady flow. Journal of Magnetic Resonance Imaging 5(4), 428-431 (1995)

15. Styner, M., et al.: Evaluation of 3D correspondence methods for model building. In: Information Processing in Medical Imaging. vol. 18 (2003) 